\title{
Lebanese Registry in the Management of Antibiotic Associated Diarrhea in Children: Observational Study in Daily Practice
}

\author{
Elie Choueirya, c, Raymond Kamel ${ }^{\mathrm{b}}$, Hilda Chacar ${ }^{\mathrm{b}}$, Pierre Mouawad ${ }^{\mathrm{b}}$, \\ Robert Sacy ${ }^{\mathrm{b}}$
}

\begin{abstract}
Background: Antibiotic-associated diarrhea (AAD) is a common complication in patients prescribed antibiotics, and represents an economic and health burden. Evidence that probiotics may be beneficial for the prevention of AAD is increasing. The aims of this registry were to assess the prevalence of probiotic prescriptions in pediatric patients for whom an antibiotic treatment regimen was prescribed, and to explore the potential health benefits that such an administration may provide.
\end{abstract}

Methods: This longitudinal, multicenter, observational study enrolled 249 pediatric patients prescribed an antibiotic treatment for 5 - 14 days, with or without a concomitant probiotic. The number of probiotic-administered patients, and AAD incidence rates throughout the 15-day follow-up period, were assessed.

Results: Of the 246 patients who met inclusion/exclusion criteria in Lebanon, the investigators had prescribed an additional probiotic treatment to $118(48 \%)$ of them, while, the other $128(52 \%)$ did not receive such additional treatment. A significantly higher number of patients in the probiotic group were at high risk of developing diarrhea (probiotic: $27.1 \%$ vs. no probiotic: $6.3 \%$; $\mathrm{P}<0.001$ ). Among high risk patients, the frequency of diarrhea was doubled in the group with no probiotics (probiotic: $21.9 \%(\mathrm{n}=7)$ vs. no probiotic: $50.0 \%$ $(n=4) ; P=0.182)$. Despite the significantly larger number of probiotic-administered patients that were at high risk of developing diarrhea, the proportion of patients who reported developing diarrhea was not statistically different between the two groups (probiotic: $\mathrm{n}=22$ $(18.6 \%)$ vs. no probiotic: $\mathrm{n}=24(18.8 \%) ; \mathrm{P}=0.983)$.

Conclusions: In conclusion, this Lebanese disease registry demonstrated that almost half of pediatric patients with mild to moderate infections were prescribed probiotics in combination with antibiotics to decrease the risk of AAD. This observation was particularly significant in the high risk population as per the treating physician's

Manuscript submitted January 19, 2017, accepted May 30, 2017

${ }^{\mathrm{a} C e n t r e ~ H o s p i t a l i e r ~ e t ~ U n i v e r s i t a i r e ~ H o t e l, ~ D i e u ~ d e ~ F r a n c e, ~ B e i r u t, ~ L e b a n o n ~}$ bSaint Georges University Hospital, Lebanon

${ }^{c}$ Corresponding Author: Elie Choueiry, Centre Hospitalier et Universitaire Hotel, Dieu de France, Beirut, Lebanon. Email: eliechoueiry@hotmail.com

doi: https://doi.org/10.14740/ijcp269w judgment.

Keywords: Pediatric antibiotic-associated diarrhea; Probiotics; $\mathrm{Ba}$ cillus clausii; Clostridium difficile

\section{Introduction}

Antibiotic-associated diarrhea (AAD), defined as an otherwise unexplained watery bowel movement that occurs in association with antibiotic use, is a common side effect of most classes of antibiotics, and is especially prevalent for broad-spectrum antibiotics [1]. Incidence rates of AAD may vary from $5 \%$ to $39 \%$, contingent on multiple factors including class of antibiotic prescribed, age of patient, environment in which the treatment is administered, and the host's general health [2-5]. The severity of AAD also tends to vary; it can manifest as a "nuisance diarrhea" with no additional complications, or as Clostridium difficile facilitated pseudomembranous colitis, a serious and progressive gastrointestinal (GI) disorder $[1,6]$. The clinical manifestation of AAD may result in prolonged hospital stays, thereby leading to significant healthcare costs. Preventive and corrective actions currently utilized for AAD include switching to an antibiotic with a narrower spectrum, or an outright cessation of antibiotic treatment, leading to partial treatment courses as well as difficulties in treating the underlying infection. No effective pre-emptive measures are currently available.

The causes of AAD are relatively well understood and characterized [7]. Studies have shown that the use of antibiotics may cause a disruption in the composition and function of the normally occurring GI microflora. Such events may result in altered carbohydrate metabolism and antimicrobial activity in the colon. Moreover, administration of antibiotics may result in allergic and toxic effects to the intestinal mucosa, thereby making the host vulnerable to the toxigenic effects of pathogenic microorganisms $[8,9]$. Such dysbiosis can arise following administration of an antibiotic that is active against anerobes, and is especially frequent following treatment with cephalosporins, clindamycin, aminopenicillins among others [1, 10-12].

Probiotics are living organisms that can produce a beneficial effect when administered in sufficient quantities [13]. Given that AAD is primarily due to a dysbiosis in GI mi- 
croflora, oral administration of probiotics may potentially rectify such an effect, thereby minimizing the risk of AAD. The potential therapeutic effects of probiotics in antibioticadministered patients have been extensively investigated over the past several years [14-25]. Evidence that administration of probiotics is beneficial for a wide range of GI disorders, including acute diarrhea associated with antibiotic use, is increasing $[14,26]$. Furthermore, two meta-analyses published in 2006 have shown that the use of probiotics was associated with a reduced risk of succumbing to AAD [27, 28]. More recently, meta-analyses by Videlock and Cremonini and Hempel et al corroborated these findings, and concluded that probiotics may present an effective preventive measure for AAD [29, 30].

The effect of probiotic therapy in the prevention of AAD in children is less well understood, as the majority of clinical trials were conducted on adult patients. Furthermore, findings from investigations conducted on these patients cannot be extrapolated to children given the conflicting data, suggesting a possible differing response in younger patients [31]. However, several studies have assessed the preventive effects of probiotics in children, and a meta-analysis by Szajewska et al has concluded that such treatment was an effective measure for minimizing AAD [9]. A more comprehensive understanding of the effect of probiotics in the prevention of AAD is needed, given that children are among the primary consumers of antibiotics [32].

The aims of this registry were to assess, for the first time, the prevalence of probiotic prescriptions in Lebanese pediatric clinical practice for children who are prescribed antibiotics, and to evaluate their efficacy and tolerability. Potential differences (demographics or clinical), between those prescribed probiotics to those who were not, were also explored.

\section{Methods}

\section{Study participants}

This study recruited male and female infants between 6 months and 12 years of age meeting the following inclusion criteria: presenting with mild to moderate infections (otitis, upper and lower respiratory tract infections, pharyngitis, urinary tract infections, etc.); had antibiotic prescription duration of 5 - 14 days. Informed consent was obtained in writing during the screening visit. Patients were excluded if: they were diagnosed with chronic diseases that may interfere with study variables (Crohn's disease, hemorrhagic recto-colitis, irritable bowel syndrome, etc.); were suffering from severe malnutrition and/ or are immuno-compromised; had an antibiotic prescription within 3 weeks of study initiation; presented with diarrhea at screening; currently taking laxatives, anti-diarrheal therapies, probiotic containing therapies/milk, drugs with adsorbing properties, or any other drug that may modify intestinal secretions and/or intestinal motility.

\section{Study design and data collection}

This longitudinal, multicenter, non-interventional, observa- tional study was scheduled to recruit 250 pediatric patients prescribed an antibiotic treatment for 5 - 14 days, with or without a concomitant probiotic. Subject eligibility was confirmed before enrollment to the study. The study consisted of one visit, followed by a longitudinal follow-up visit or phone contact $2-15$ days ( \pm 1 day) later. The enrollment period was 6.5 months. Patient demographics, vital signs, medical history, type of infection, and treatment strategy were assessed for all patients during the initial visit. Subject weight, concomitant medications, and treatment compliance were assessed during the follow-up visit, and a stool sample was obtained when possible.

\section{Study objectives}

The primary objective for this registry was to assess the frequency of probiotic prescription in patients for whom an antibiotic was prescribed. Secondary objectives were as follows: summarize the demographic and clinical characteristics of patients for whom a supplementary probiotic was prescribed; proportion of "high risk" patients prescribed a probiotic treatment regimen; proportion of patients who develop diarrhea in both groups; bacteria identified following analysis of patients' stool samples when performed. Risk of developing AAD was determined by the type of antibiotic used, previous history of AAD and age of participants.

Safety analyses included assessing the incidence and frequency of adverse event (AE) and serious adverse event (SAE), the severity of reported events, and the relationship between such events and the study medication.

\section{Statistical analysis}

A sample size of 250 should allow for an estimation of the prevalence of probiotic prescription, assumed to be $50 \%$, with a precision not exceeding $\pm 6.2 \%$ using a $95 \%$ confidence interval (CI). Furthermore, if patient distribution among the two cohorts (with probiotics vs. no probiotics) is similar, we should be able to detect differences of magnitude of $20 \%$ or more (17.5\% or more) in any demographic or clinical characteristic between the two groups with $90 \%$ power $(80 \%$ power respectively) using the Chi-squared test.

Baseline descriptive analyses were carried out for all eligible subjects who had met the inclusion/exclusion criteria of the study. All eligible subjects who had not committed protocol violations, and have at least one evaluable study end point variable (probiotic prescription) comprised the analysis population, and were used in the efficacy analysis. Student's $t$-test was used for comparisons between groups of patients (with and without a probiotic prescription) regarding age (years), basal weight/weight change $(\mathrm{kg})$, oral temperature $\left({ }^{\circ} \mathrm{C}\right)$, heart rate (beats/min), antibiotic duration (days), and mean number of stools per day. Differences in gender, antibiotic type, risk of developing diarrhea, type of infection and hospitalization were assessed using Chi-square test. Chi-square test was also used to compare the proportion of patients who developed diarrhea in the follow-up period. Comparisons between groups regarding the most frequently identified bacteria in patients' stool 


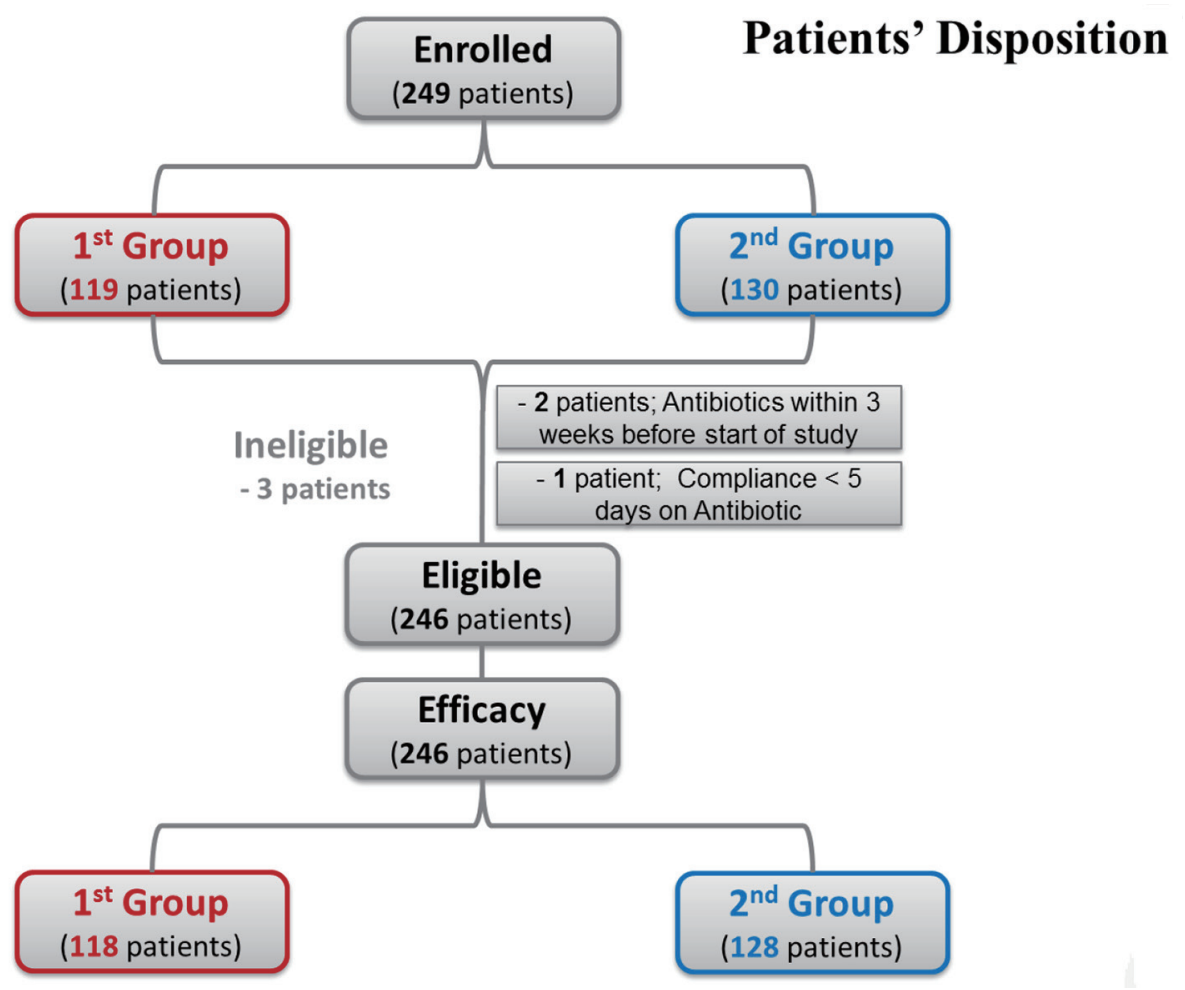

Figure 1. Flowchart of patient enrollment.

samples were carried out using the Chi-square test.

Binary logistic regression was used to identify patients' odds of receiving a concomitant probiotic alongside the initial antibiotic treatment, and the prospective factors that were assessed included the age of the subject, gender, risk of developing diarrhea, infection type, antibiotic class used, and duration of antibiotic prescription. Binary logistic analysis was also conducted to determine whether age was a significant predictor of diarrheal disease in children administered an antibiotic treatment regimen.

\section{Ethical considerations}

This registry was conducted in accordance with the principles established by the 18th World Medical Assembly (Helsinki, 1964) and all applicable amendments laid down by it, as well as the ICH guidelines for good clinical practice. This study was conducted in compliance with all national and international laws and regulations. Written informed consent was obtained from all participants' parents in this study.

\section{Results}

\section{Patient status and characteristics}

A flow diagram of the current study is shown in Figure 1. A total of 249 patients were recruited for screening. Of those, 247 met inclusion/exclusion criteria; two patients were on antibiotics within 3 weeks of study commencement and were therefore excluded. One patient committed a protocol violation; patient was not compliant with antibiotic prescription $(<5$ days $)$. The remaining 246 patients were included in the efficacy analysis, and the physician-prescribed treatment regimen was assessed. The first group of the study encompassed 118 patients (48\%) prescribed an antibiotic/probiotic dual therapy, while the second group included 128 patients $(52 \%)$ who received antibiotics alone (Fig. 2).

The clinical characteristics of study participants are highlighted in Table 1 and Figure 3. There were no significant differences among the two groups regarding mean age, gender, vital signs, disease characteristics, and the class of antibiotics prescribed. Furthermore, there were no significant differences in the antibiotic prescription duration between the two groups (probiotic: $9.17 \pm 1.56$ vs. no probiotic: $9.04 \pm 1.56 ; \mathrm{P}=0.473$; Table 1). The majority of patients were prescribed an antibiotic for the upper respiratory tract infections (URTIs) $(\mathrm{n}=112$, $45.5 \%)$, and otitis $(n=97,39.4 \%$; Fig. 3c). The most commonly prescribed antibiotics included penicillins (probiotic: $n=76$ (64.4\%) vs. no probiotic: $\mathrm{n}=80(62.5 \%))$ and cephalosporins (probiotic: $n=33(28.0 \%)$ vs. no probiotic: $n=42(32.8 \%)$; Fig. 3d). Three (1.2\%) out of 246 eligible subjects were noncompliant with their allocated antibiotic treatments for multiple reasons. The reasons for non-compliance include a change in the antibiotic prescribed as reported by two patients $(0.8 \%)$; lack of treatment efficacy was reported by one patient $(0.4 \%)$ and parent's non-compliance for another patient $(0.4 \%)$. On the other hand, all patients in the probiotic group were compli- 


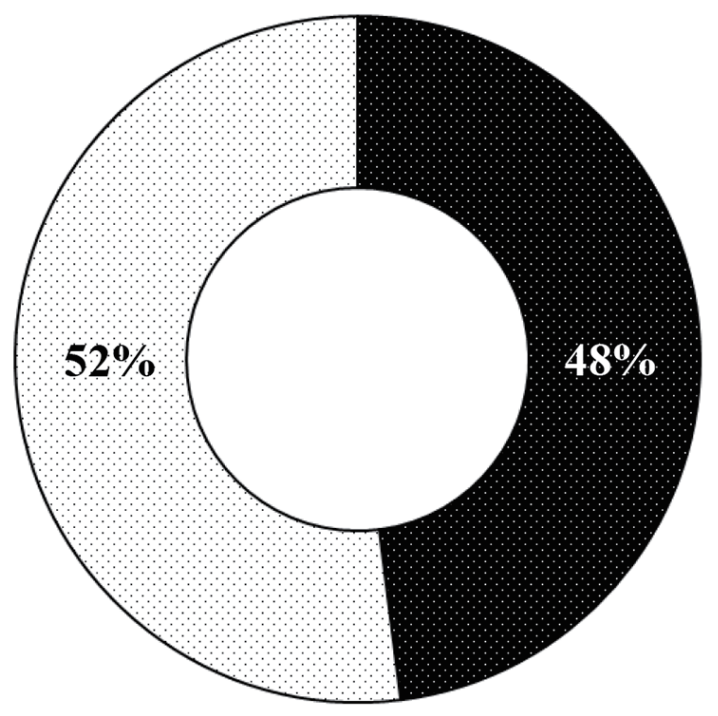

Probiotics Prescribed $\square$ Probiotics not Prescribed

Figure 2. Prevalence of probiotic prescriptions in the study population.

Table 1. Baseline Characteristics of Study Participants

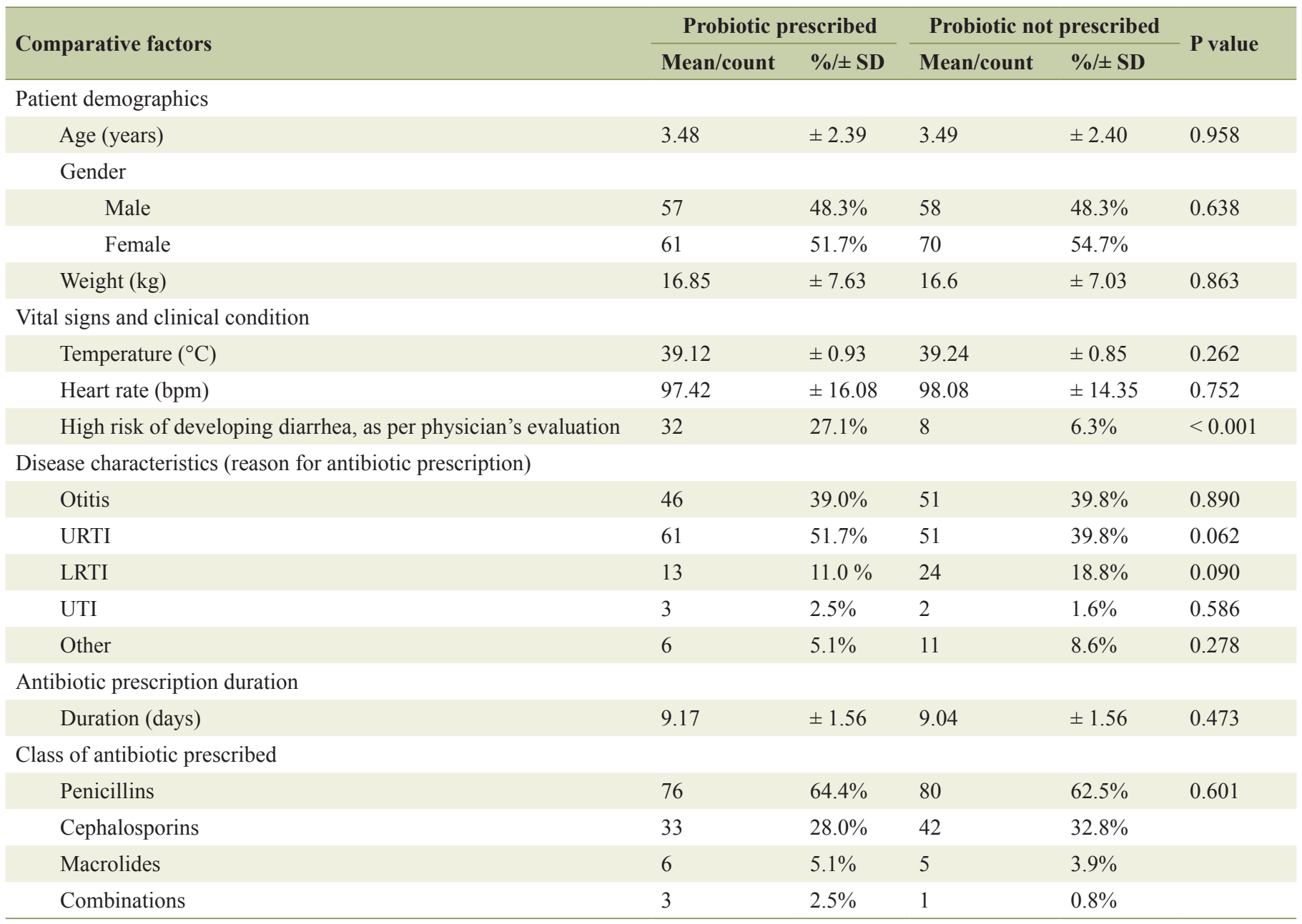

URTI: upper respiratory tract infection; LRTI: lower respiratory tract infections; UTI: urinary tract infections. 

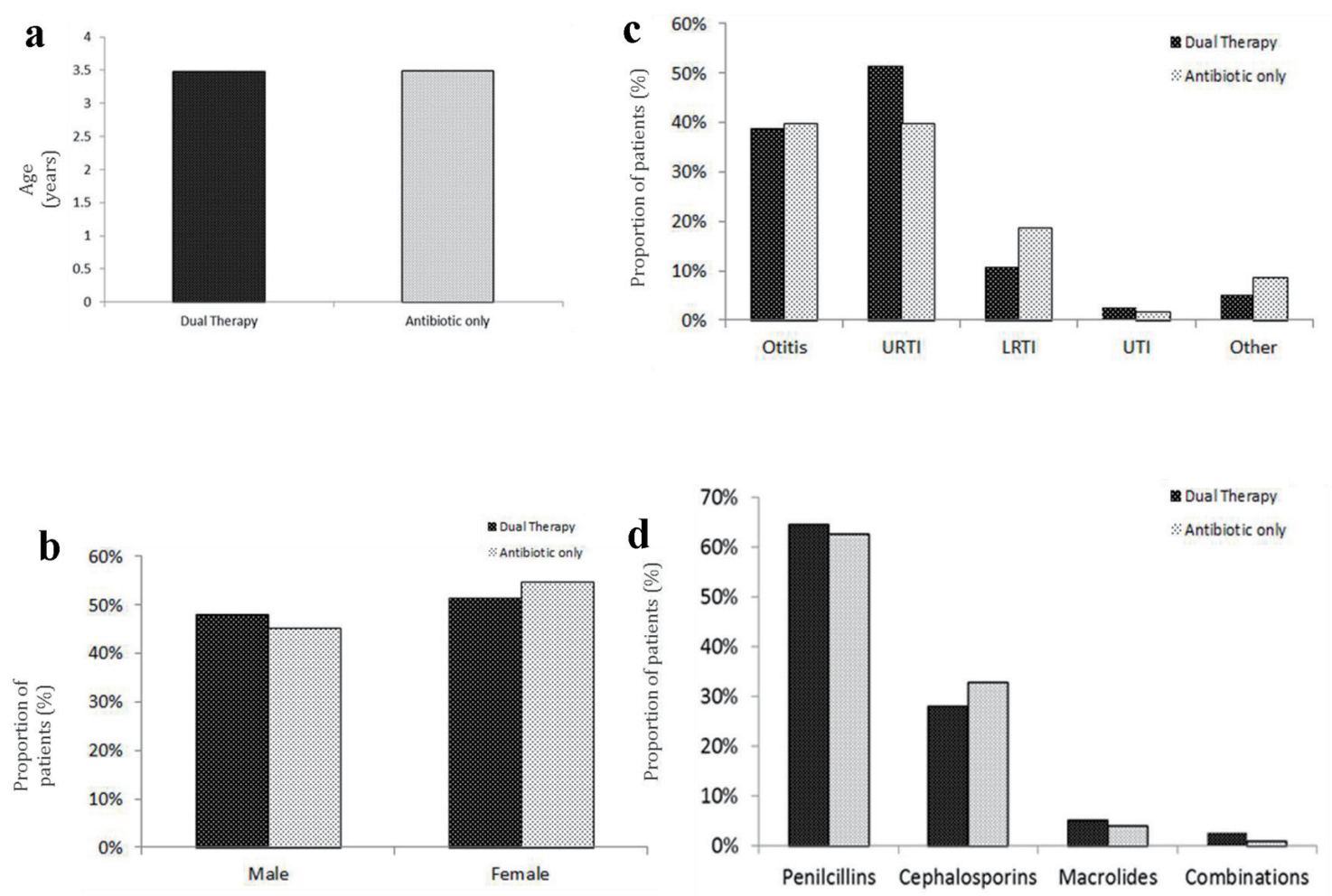

Figure 3. Baseline characteristics of the study population: (a) mean age; (b) gender distribution; (c) type of infection treated; (d) type of antibiotic administered. URTI: upper respiratory tract infection; LRTI: lower respiratory tract infection; UTI: urinary tract infection.

ant to their prescribed probiotics.

\section{Primary endpoint}

\section{Probiotic prescriptions}

Of the 246 enrolled subjects for whom an antibiotic treatment regimen was prescribed, 118 (48\%; 95\% CI (41.7-54.3\%)) were administered a supplementary course of probiotics as a means of preventing any AAD (Fig. 2, Table 2). The most frequently prescribed probiotic was Bacillus clausii $(\mathrm{n}=96$ (81.4\%); Fig. 4). Other commonly utilized probiotics included a combination of Lactobacillus acidophilus and Bifidobacterium lactis $(\mathrm{n}=7(5.9 \%))$, Lactobacillus acidophilus $(\mathrm{n}=4$
(3.4\%)) and Lactobacillus acidophilus/Bifidobacterium breve combinations $(\mathrm{n}=4(3.4 \%))$. Prescribed probiotics were commercial tablets/capsules or liquid containing microorganisms and they were prescribed for an average of 10 days. Patients prescribed a probiotic were compliant with their treatment.

\section{Secondary endpoints}

Demographic and clinical characteristics of probiotic-administered patients

Prescription of a probiotic was not significantly associated with age ( $\mathrm{P}=0.958)$, gender $(\mathrm{P}=0.638)$, weight $(\mathrm{P}=0.863)$, class of antibiotic treatment prescribed $(\mathrm{P}=0.601)$, and the in-

Table 2. Prevalence of Probiotic Prescription and the Clinical Condition of Study Participants

\begin{tabular}{llll} 
& \multicolumn{2}{c}{ Method of treatment } \\
\cline { 2 - 4 } & Probiotic prescribed & Probiotic not prescribed \\
\hline Number of subjects (\%) & $118(48 \%)$ & $128(52 \%)$ & $-/-$ \\
$\begin{array}{l}\text { Number of watery stools experienced by AAD } \\
\text { afflicted patients 24 h prior to follow-up examination }\end{array}$ & $3.65 \pm 1.64$ & $4.74 \pm 2.61$ \\
Mean change in weight (kg) & $+0.044 \pm 0.255$ & $-0.049 \pm 0.394$ & 0.100 \\
Mean age of patients experiencing diarrhea & $2.50 \pm 1.65$ & $3.71 \pm 2.47$ & 0.253 \\
\hline
\end{tabular}




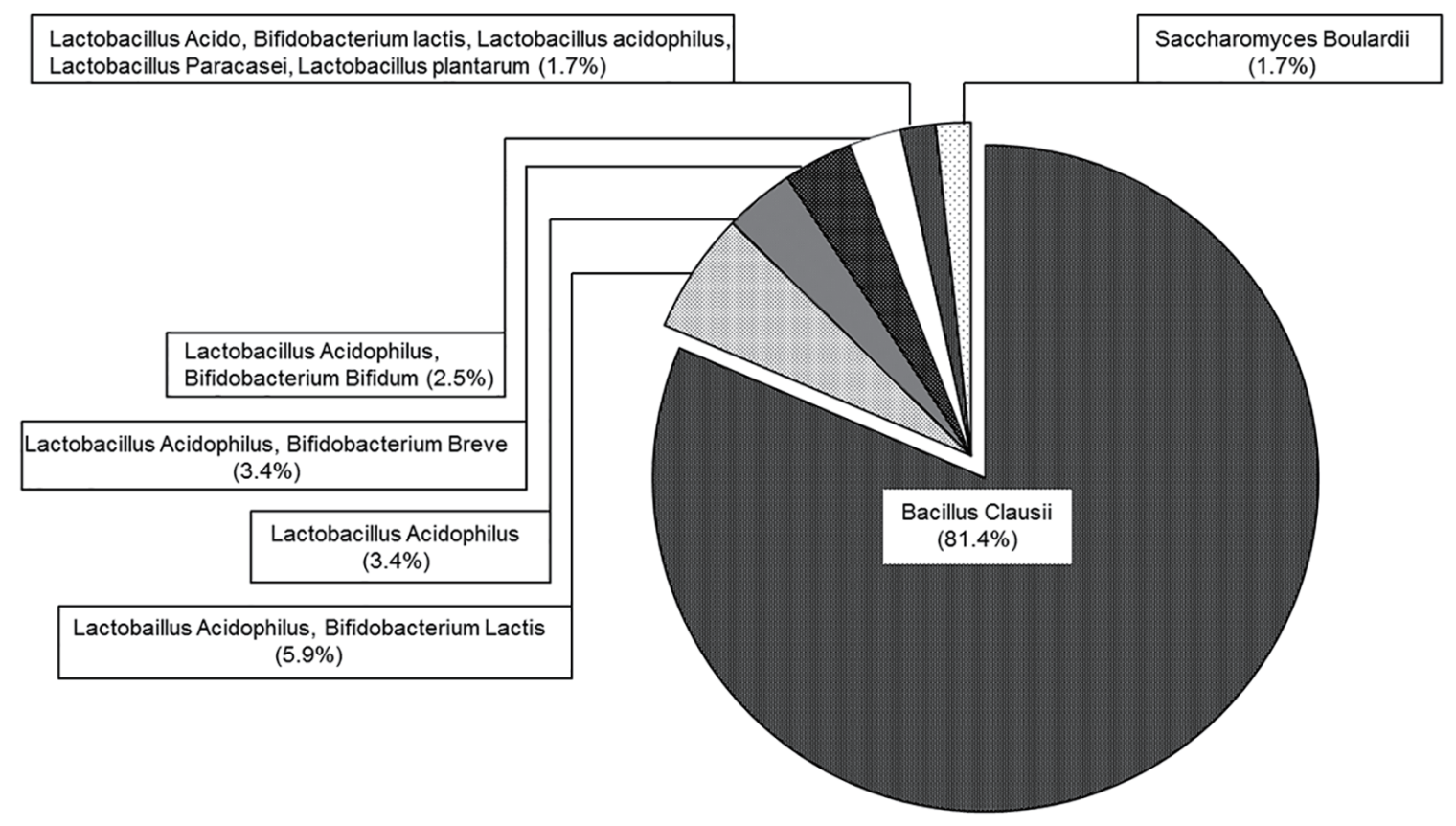

Figure 4. Probiotic prescriptions. Relative proportions of probiotics administered to enrolled subjects receiving such treatment.

fection type (Table 1). However, a significantly higher number of patients at high risk of developing AAD, as evaluated by treating physician, were prescribed a supplementary probiotic for the prevention of AAD (probiotic: $n=32(27.1 \%)$ vs. no probiotic: $\mathrm{n}=8(6.3 \%)$; $\mathrm{P}<0.001$; Fig. 5a, Table 1).

When comparing baseline characteristics among patients with and without probiotic prescription, no significant associations were observed in regards to the age, gender, type of infection, class of antibiotic prescribed and the duration of antibiotic treatment. However, there was a significant association between the risk of developing diarrhea and probiotic prescription. Namely, prescription of probiotics was about seven-fold higher in patients at high risk of developing AAD $(\mathrm{P}<0.001)$.

\section{Incidence of $A A D$}

Despite the significantly larger number of probiotic-administered patients that were at high risk of developing diarrhea, the proportion of patients who reported developing diarrhea was not statistically different between the two groups (probiotic: $\mathrm{n}=22(18.6 \%)$ vs. no probiotic: $\mathrm{n}=24(18.8 \%) ; \mathrm{P}=$ 0.983; Fig. 5c). When looking exclusively at the 40 patients who were classified as high risk, a lower (but not significant) percentage of patients in the probiotic group developed diarrhea (probiotic $n=7(21.9 \%)$ vs. no probiotic $n=4(50 \%), P$ $=0.182$; Fig. 5b).

Patients experiencing AAD were of a significantly younger age when compared to those who did not $(2.5 \pm 1.65$ years vs. $3.7 \pm 2.47$ years; $\mathrm{P}=0.001)$. However, binary logistic regression analyses revealed no significant predictive value for age in diarrhea occurrence when controlling for other factors (gender, risk of developing diarrhea, infection type, antibiotic class used, and duration of antibiotics/probiotics prescription).

\section{Microbiota identified in stool analyses}

A total of 46 subjects reported experiencing diarrhea. Stool samples for analysis were provided from three patients only (two patients from the probiotic group, and one patient from the second group). Microbiological sub-analyses of the samples revealed the presence of the Candida spp. fungus in both patients for whom probiotics were prescribed (data not shown). As for the patient not prescribed a probiotic, the sub-analysis revealed no abnormal growth.

\section{Weight changes and frequency of watery stools}

No significant differences between both groups of patients, in regards to the mean change in weight, were observed (probiotic: $+0.044 \pm 0.255 \mathrm{~kg}$ vs. no probiotic: $-0.049 \pm 0.394 ; \mathrm{P}=$ 0.253 ; Fig. 6a, Table 2). Furthermore, no statistically significant differences in the patients reporting having experienced watery stools, $24 \mathrm{~h}$ prior to the follow-up visit, were noted (probiotic $=3.65 \pm 1.64$ episodes, no probiotic $=4.74 \pm 2.61$ episodes; $\mathrm{P}=0.10$; Fig. $6 \mathrm{~b}$, Table 2 ).

\section{Safety and tolerability}

Diarrhea and associated symptoms were captured in the CRF as part of the study endpoints. However, no AEs associated 
a Pediatric Patients at High Risk to Develop Diarrhea

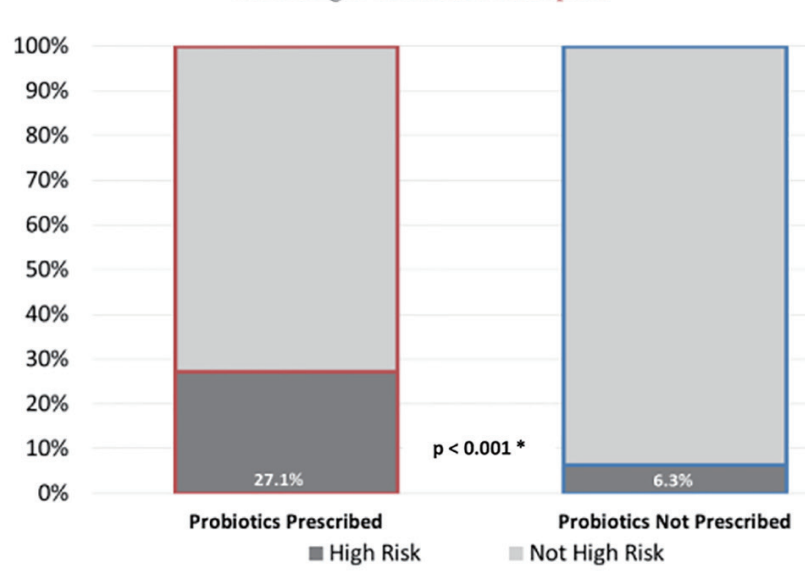
b High Risk Patients who Developed Diarrhea among patients With Probiotic Prescription and those Without

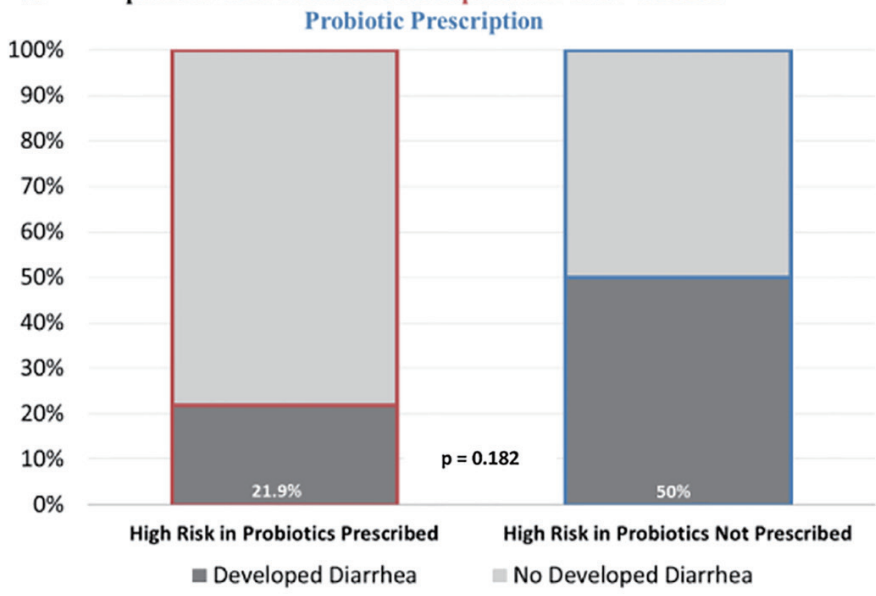

C Pediatric Patients who Developed Diarrhea among patients With Probiotic Prescription and those Without Probiotic Prescription

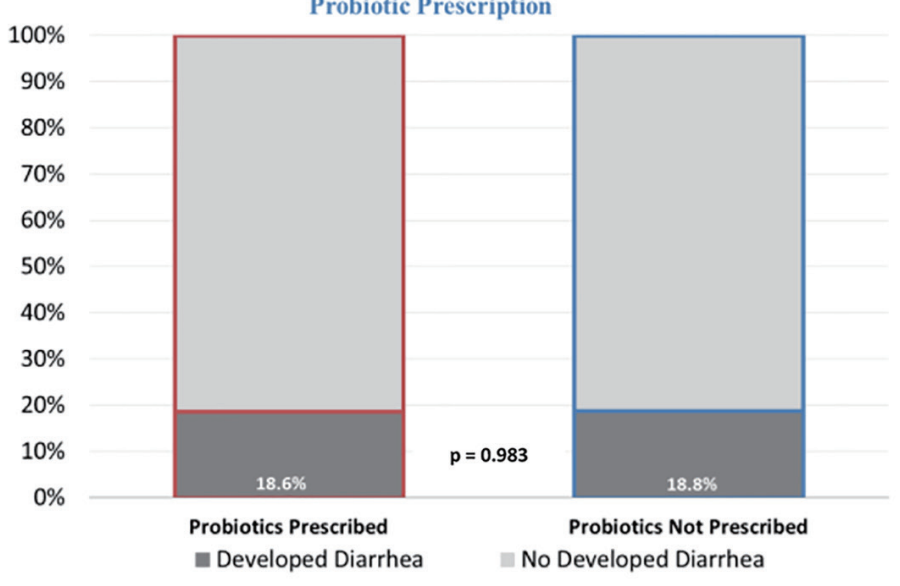

Figure 5. Prevalence of diarrhea in the study population. (a) Proportion of patients classified as "high risk" by the evaluating physician. (b) The number of patients classified as "high risk" presenting with AAD throughout the study period. (c) The overall number of patients exhibiting AAD throughout the study period. 

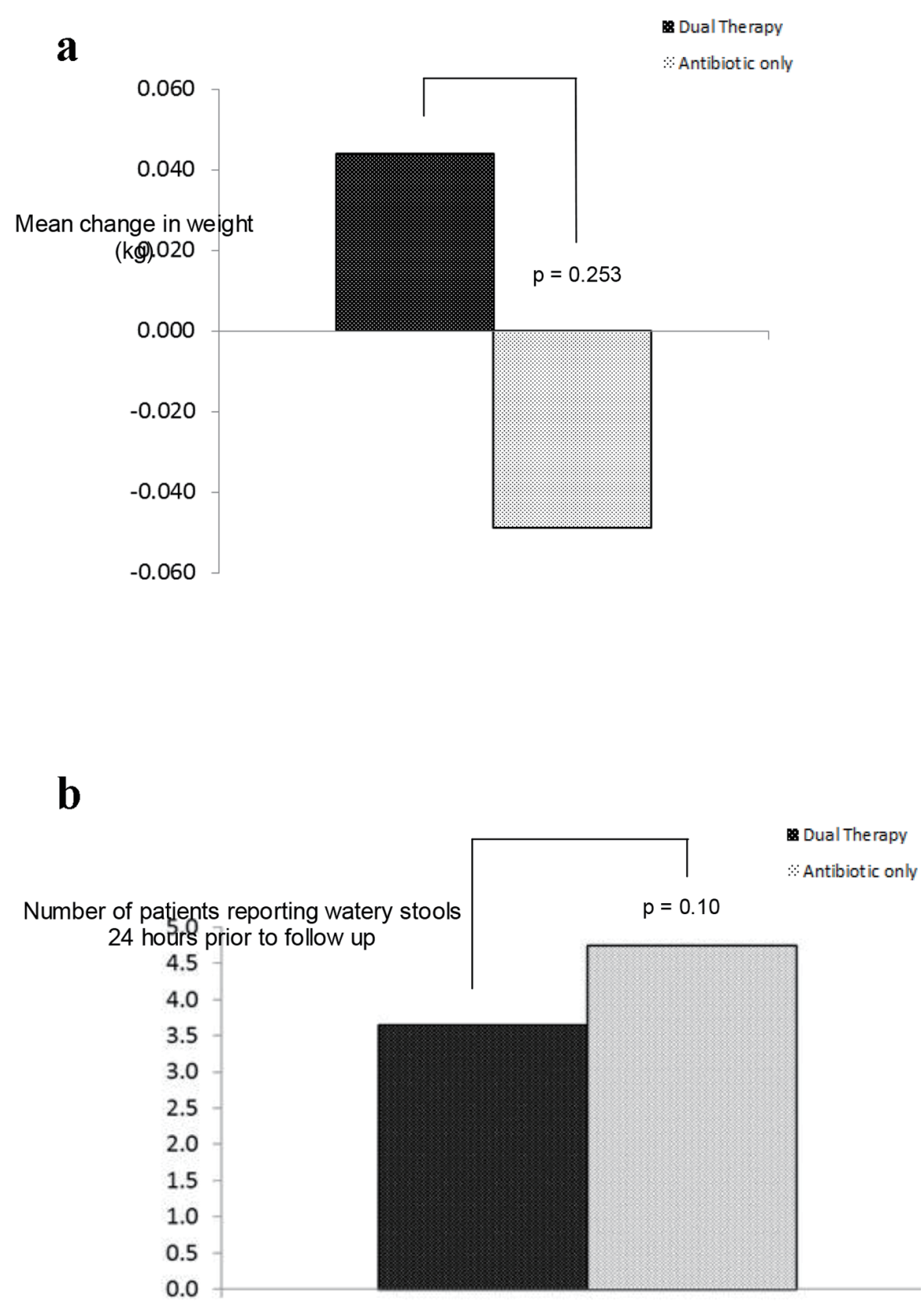

Figure 6. Incidence of diarrhea: (a) mean change in weigh; (b) number of patients experiencing diarrhea $24 \mathrm{~h}$ prior to follow-up.

with the use of probiotics were reported.

\section{Discussion}

This multicenter, prospective, observational, non-comparative, 15-day disease registry was conducted in 24 sites in Lebanon (Fig. 7). A total of 249 pediatric patients, aged between 6 months and 12 years, for whom a 5 - 14 days antibiotic course was prescribed to treat mild to moderate infections, were recruited. The primary objective of the current registry was to assess the prevalence of probiotic use in pediatric patients for whom an antibiotic treatment course has been prescribed as per investigators' sole discretion. Approximately half of the subjects who have participated were prescribed a supplementary probiotic by their physician. Of patients not prescribed a probiotic, $60.2 \%$ were administered alternative prevention measures by their physician, primarily involving dietary changes. Moreover, $25 \%$ were not assigned a prescription as they were deemed to be at low risk of developing treatment-associated diarrhea, while $8.6 \%$ did not receive a prescription since it was not part of their physician's routine practice. 


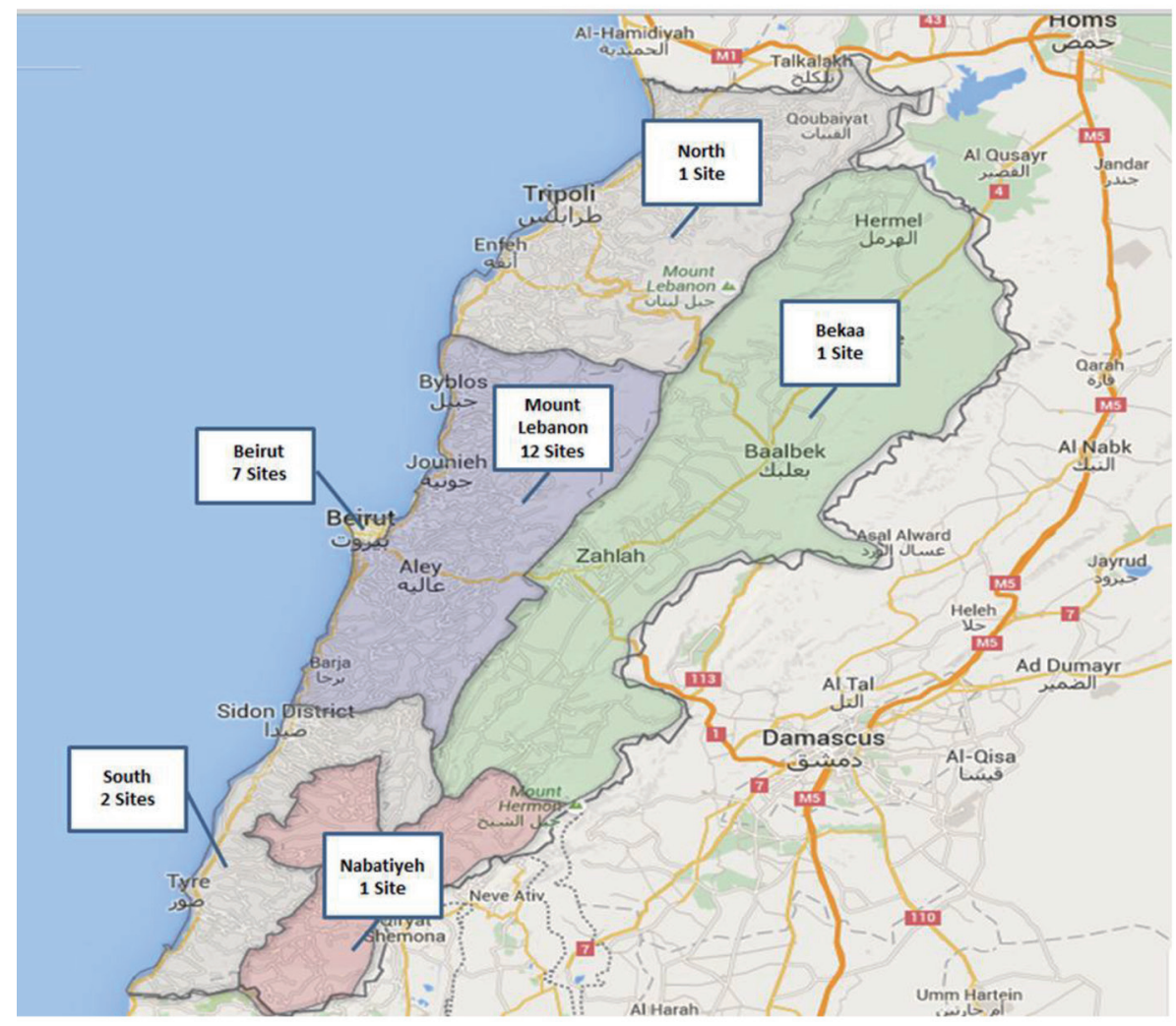

Figure 7. Map depicting the location of sites from which patients were recruited.

There is growing evidence that the prophylactic administration of probiotics is an effective measure for preventing AAD. In two recent meta-analyses, Videlock et al and Hempel et al agreed that the prophylactic administration of probiotics resulted in a reduced risk of AAD [29, 30]. The World Gastroenterology Organization (WGO) guideline now states that there is a strong evidence of efficacy, and recommends the use of probiotics in clinical practice [13].

It is of note that a significant association between the risk of developing diarrhea and probiotic prescription was observed. Namely, patients deemed to be at high risk of developing AAD were more likely to be prescribed a supplementary probiotic treatment $(27.1 \%$ probiotic group vs. $6.3 \%$ non-probiotic group; $\mathrm{P}<0.001)$. However, this disparity between the two groups did not translate into incidence of AAD; no significant differences between the two groups were observed. It is important to highlight that the study sample size was calculated to primarily assess the prevalence of probiotic prescriptions among pediatric patients for whom antibiotics were prescribed, and not to compare the efficacy of probiotics between two groups. Therefore, comparative efficacy analyses are beyond the scope of our current investigation. A different study design and sample size is required to assess the efficacy of probiotics.

It is also noteworthy that $\mathrm{AAD}$ incidence rates in this study were comparable to those previously reported [33, 34]. Patients presenting with treatment-induced diarrhea were significantly younger than those who did not (diarrhea: $2.50 \pm$ 1.65 years vs. no diarrhea: $3.71 \pm 2.47$ years), also consistent with previous findings where younger patients appeared to be at a higher risk of developing $\mathrm{AAD}[9,35]$.

Clostridium difficile is notorious for its role in $\mathrm{AAD}$, and in a study by Arvola et al, stool samples from pediatric patients presenting with AAD tested positive for this pathogen [36]. However, studies have shown that Clostridium difficile only accounts for $10-20 \%$ of AAD cases [37-39], indicating that other pathogens may be involved. The provision of a stool sample was entirely optional in our study, and analyses of these samples were conducted to identify the most common pathogens in patients who develop diarrhea. Such analyses would have been of clinical significance, and shed light on the enteric microorganisms involved in pediatric AAD. However, only three patients provided a sample during the follow-up visit. Two stool samples tested positive for the Candida spp. fungus, and no abnormal growth was detected for the third. Given the low participation rates, no reliable conclusions can be drawn from this data set.

No AEs, including serious ones, were reported in this investigation. It is well established that probiotics are well tolerated in patients, and they have been extensively used for decades. Most clinical trials involving the use of probiotics to prevent AAD and Clostridium difficile-associated diarrhea (CDAD) have not reported serious adverse reactions [4]. Moreover, in a recent systematic review addressing the safety of probiotics, no significant differences in the overall number of AE/SAEs were observed and short-term probiotic use was recommended. 
There are some limitations to the current study that should be addressed. Given the observational nature of the study, confounding and selection bias may have influenced our findings. The lack of significant differences in the incidence of AAD for the two groups may be a result of the limited number of participants that were enrolled. It is also important to highlight the relatively short, 15-day follow-up period. According to previous reports, it is crucial for studies assessing AAD to have sufficiently long follow-up periods, as the manifestation of AAD may be delayed by up to 4 - 6 weeks following the cessation of antibiotic treatment regimens [2, 33, 34, 40]. It is therefore possible that the lack of significant differences between the two cohorts, in regards to the incidence of treatment-associated diarrhea, was a consequence of the short follow-up period employed for this registry. In a study with a similarly short follow-up period (15 days), Lewis et al were unsuccessful at demonstrating any significant reduction in AAD incidence in elderly patients following the administration of Saccharomyces boulardii [31]. However, in another study assessing the potential effect of Saccharomyces boulardii in preventing AAD in children, a significant beneficial effect was observed despite the short, 2-week follow-up period [41].

The observational nature of the study also means that probiotic and antibiotic prescriptions were administered to patients at the physician's discretion. As a result, patients were administered a wide variety of probiotics, and correlative analyses assessing the relationship between the probiotic received and the incidence of AAD would not be effective. Different probiotic strains have been shown to act differently in in vitro studies [42], and the most suitable strain of probiotic for the prevention of $\mathrm{AAD}$ is yet to be unequivocally determined [16, 20, 43-45]. Identifying probiotic strains that most effectively prevent AAD remains an area of future research, and additional comparative studies are needed.

\section{Conclusions}

In summary, our results show that among pediatric patients with mild to moderate infections for whom antibiotics were prescribed, almost half were prescribed a prophylactic probiotic to prevent AAD. Patients considered to be at higher risk are more likely to be administered a probiotic supplement. It is important to highlight that the study sample size was calculated to address the primary study objective of estimating the prevalence of probiotic prescription among the pediatric patients for whom antibiotics were prescribed and not to compare the efficacy of probiotics between two groups. A different study design and sample size is required to assess the efficacy of probiotics.

\section{Acknowledgments}

This investigation was conducted by hospital- and clinic-based physicians from 24 sites across Lebanon; 12 sites in Mount Lebanon, seven sites in the capital Beirut, two sites in southern Lebanon, and a site each in northern Lebanon, Nabatiyeh, and
Bekaa. The study was sponsored and funded by Sanofi-aventis Liban SAL, Lebanon, which played no role in analyzing or interpreting the data. Statistical analyses and editorial support was provided by Mohamed Taha and Kareem S. El Iskandarani of RAY contract research organization, and was funded by Sanofi.

\section{Conflicts of Interest}

The authors of this study report no conflicts of interest.

\section{List of Investigators}

Elie Choueiry, Maroun Matar, Pierre Mouawad, Robert Sacy, Raymond Kamel, Hilda Chacar, Imad Chokr, Alia Al Araj, Ali Zeitoun, Hassan Kobeissi, Youssef Francis, Marie-Claude Khalifeh, Ziad Bassil, Kamal Saad, Alain Sayad, Varouj Margossian, Maroun Sokhen, Imad Khayat, Antoine Yazbeck, Bassam Ghanem, Khalil Osta, Mohamad Zbib, Julien Lteif, Chaiban Raffoul.

\section{Abbreviations}

AAD: antibiotic-associated diarrhea; AE: adverse event; CDAD: Clostridium difficile-associated disease; CI: confidence interval; GI: gastrointestinal; OR: odds ratio; P: probability; SAE: serious adverse event; WGO: World Gastroenterology Organization

\section{References}

1. Bartlett JG. Clinical Practice. Antibiotic-Associated Diarrhea. N Engl J Med. 2002;346(5):334-339.

2. McFarland LV. Epidemiology, Risk Factors and Treatments for Antibiotic-Associated Diarrhea. Dig Dis. 1998;16(5):292-307.

3. Surawicz CM. Antibiotic-Associated Diarrhea and Pseudomembranous Colitis: Are They Less Common with Poorly Absorbed Antimicrobials? Chemotherapy. 2005;51(Suppl 1):81-89.

4. McFarland LV. Evidence-Based Review of Probiotics for Antibiotic-Associated Diarrhea and Clostridium Difficile Infections. Anaerobe. 2009;15(6):274-280.

5. Bergogne-Berezin E. Treatment and Prevention of Antibiotic Associated Diarrhea. Int J Antimicrob Agents. 2000;16(4):521-526.

6. Bartlett JG, Chang TW, Gurwith M, Gorbach SL, Onderdonk AB. Antibiotic-Associated Pseudomembranous Colitis Due to Toxin-Producing Clostridia. N Engl J Med. 1978;298(10):531-534.

7. Hogenauer C, Hammer HF, Krejs GJ, Reisinger EC. Mechanisms and Management of Antibiotic-Associated Diarrhea. Clin Infect Dis. 1998;27(4):702-710.

8. Chung H, Kasper DL. Microbiota-Stimulated Immune 
Mechanisms to Maintain Gut Homeostasis. Curr Opin Immunol. 2010;22(4):455-460.

9. Szajewska H, Ruszczynski M, Radzikowski A. Probiotics in the Prevention of Antibiotic-Associated Diarrhea in Children: A Meta-Analysis of Randomized Controlled Trials. J Pediatr. 2006;149(3):367-372.

10. Barbut F, Meynard JL, Guiguet M, Avesani V, Bochet MV, Meyohas MC, Delmee M, et al. Clostridium Difficile-Associated Diarrhea in Hiv-Infected Patients: Epidemiology and Risk Factors. J Acquir Immune Defic Syndr Hum Retrovirol. 1997;16(3):176-181.

11. McFarland LV, Surawicz CM, Stamm WE. Risk Factors for Clostridium Difficile Carriage and C. Difficile-Associated Diarrhea in a Cohort of Hospitalized Patients. J Infect Dis. 1990;162(3):678-684.

12. Wistrom J, Norrby SR, Myhre EB, Eriksson S, Granstrom G, Lagergren L, Englund G, et al. Frequency of Antibiotic-Associated Diarrhoea in 2462 Antibiotic-Treated Hospitalized Patients: A Prospective Study. J Antimicrob Chemother. 2001;47(1):43-50.

13. FAO/WHO. Evaluation of Health and Nutritional Properties of Probiotics in Food Including Powder Milk With Live Lactic Acid Bacteria, Food and Agriculture Organization of the United Nations and World Health Organization, Joint FAO/WHO Expert Consultation Group: Cordoba, Argentina.

14. Hickson M, D'Souza AL, Muthu N, Rogers TR, Want S, Rajkumar C, Bulpitt CJ. Use of Probiotic Lactobacillus Preparation to Prevent Diarrhoea Associated with Antibiotics: Randomised Double Blind Placebo Controlled Trial. BMJ. 2007;335(7610):80.

15. Issa I, Moucari R. Probiotics for Antibiotic-Associated Diarrhea: Do We Have a Verdict? World J Gastroenterol. 2014;20(47):17788-17795.

16. Song HJ, Kim JY, Jung SA, Kim SE, Park HS, Jeong Y, Hong SP, et al. Effect of Probiotic Lactobacillus (Lacidofil(R) Cap) for the Prevention of AntibioticAssociated Diarrhea: A Prospective, Randomized, Double-Blind, Multicenter Study. J Korean Med Sci. 2010;25(12):1784-1791.

17. Cimperman L, Bayless G, Best K, Diligente A, Mordarski B, Oster M, Smith M, et al. A Randomized, Double-Blind, Placebo-Controlled Pilot Study of Lactobacillus Reuteri Atcc 55730 for the Prevention of Antibiotic-Associated Diarrhea in Hospitalized Adults. J Clin Gastroenterol. 2011;45(9):785-789.

18. Wunderlich PF, Braun L, Fumagalli I, D'Apuzzo V, Heim F, Karly M, Lodi R, et al. Double-Blind Report on the Efficacy of Lactic Acid-Producing Enterococcus Sf68 in the Prevention of Antibiotic-Associated Diarrhoea and in the Treatment of Acute Diarrhoea. J Int Med Res. 1989;17(4):333-338.

19. Gotz V, Romankiewicz JA, Moss J, Murray HW Prophylaxis against Ampicillin-Associated Diarrhea with a Lactobacillus Preparation. Am J Hosp Pharm. 1979;36(6):754-757.

20. Armuzzi A, Cremonini F, Bartolozzi F, Canducci F, Candelli M, Ojetti V, Cammarota G, et al. The Effect of Oral Administration of Lactobacillus Gg on Antibiotic-Asso- ciated Gastrointestinal Side-Effects During Helicobacter Pylori Eradication Therapy. Aliment Pharmacol Ther. 2001;15(2):163-169.

21. Cremonini F, Di Caro S, Covino M, Armuzzi A, Gabrielli M, Santarelli L, Nista EC, et al. Effect of Different Probiotic Preparations on Anti-Helicobacter Pylori Therapy-Related Side Effects: A Parallel Group, Triple Blind, Placebo-Controlled Study. Am J Gastroenterol. 2002;97(11):2744-2749.

22. Myllyluoma E, Veijola L, Ahlroos T, Tynkkynen S, Kankuri E, Vapaatalo H, Rautelin H, et al. Probiotic Supplementation Improves Tolerance to Helicobacter Pylori Eradication Therapy - a Placebo-Controlled, DoubleBlind Randomized Pilot Study. Aliment Pharmacol Ther. 2005;21(10):1263-1272.

23. Nista EC, Candelli M, Cremonini F, Cazzato IA, Zocco MA, Franceschi F, Cammarota G, et al. Bacillus Clausii Therapy to Reduce Side-Effects of Anti-Helicobacter Pylori Treatment: Randomized, Double-Blind, Placebo Controlled Trial. Aliment Pharmacol Ther. 2004;20(10):11811188.

24. Allen SJ, Wareham K, Wang D, Bradley C, Hutchings H, Harris W, Dhar A, et al. Lactobacilli and Bifidobacteria in the Prevention of Antibiotic-Associated Diarrhoea and Clostridium Difficile Diarrhoea in Older Inpatients (Placide): A Randomised, Double-Blind, Placebo-Controlled, Multicentre Trial. Lancet. 2013;382(9900):12491257.

25. Beniwal RS, Arena VC, Thomas L, Narla S, Imperiale TF, Chaudhry RA, Ahmad UA. A Randomized Trial of Yogurt for Prevention of Antibiotic-Associated Diarrhea. Dig Dis Sci. 2003;48(10):2077-2082.

26. Hoffman FA, Heimbach JT, Sanders ME, Hibberd PL. Executive Summary: Scientific and Regulatory Challenges of Development of Probiotics as Foods and Drugs. Clin Infect Dis. 2008;46(Suppl 2):S53-57.

27. McFarland LV. Meta-Analysis of Probiotics for the Prevention of Antibiotic Associated Diarrhea and the Treatment of Clostridium Difficile Disease. Am J Gastroenterol. 2006;101(4):812-822.

28. Sazawal S, Hiremath G, Dhingra U, Malik P, Deb S, Black RE. Efficacy of Probiotics in Prevention of Acute Diarrhoea: A Meta-Analysis of Masked, Randomised, Placebo-Controlled Trials. Lancet Infect Dis. 2006;6(6):374382.

29. Videlock EJ, Cremonini F. Meta-Analysis: Probiotics in Antibiotic-Associated Diarrhoea. Aliment Pharmacol Ther. 2012;35(12):1355-1369.

30. Hempel S, Newberry SJ, Maher AR, Wang Z, Miles JN, Shanman R, Johnsen B, et al. Probiotics for the Prevention and Treatment of Antibiotic-Associated Diarrhea: A Systematic Review and Meta-Analysis. JAMA. 2012;307(18):1959-1969.

31. Lewis SJ, Potts LF, Barry RE. The Lack of Therapeutic Effect of Saccharomyces Boulardii in the Prevention of Antibiotic-Related Diarrhoea in Elderly Patients. J Infect. 1998;36(2):171-174.

32. Sommet A, Sermet C, Boelle PY, Tafflet M, Bernede C, Guillemot D. No Significant Decrease in Antibiotic Use 
from 1992 to 2000, in the French Community. J Antimicrob Chemother. 2004;54(2):524-528.

33. Turck D, Bernet JP, Marx J, Kempf H, Giard P, Walbaum $\mathrm{O}$, Lacombe A, et al. Incidence and Risk Factors of Oral Antibiotic-Associated Diarrhea in an Outpatient Pediatric Population. J Pediatr Gastroenterol Nutr. 2003;37(1):2226.

34. Elstner CL, Lindsay AN, Book LS, Matsen JM. Lack of Relationship of Clostridium Difficile to AntibioticAssociated Diarrhea in Children. Pediatr Infect Dis. 1983;2(5):364-366.

35. Cruchet S, Furnes R, Maruy A, Hebel E, Palacios J, Medina F, Ramirez N, et al. The Use of Probiotics in Pediatric Gastroenterology: A Review of the Literature and Recommendations by Latin-American Experts. Paediatr Drugs. 2015;17(3):199-216.

36. Arvola T, Laiho K, Torkkeli S, Mykkanen H, Salminen S, Maunula L, Isolauri E. Prophylactic Lactobacillus Gg Reduces Antibiotic-Associated Diarrhea in Children with Respiratory Infections: A Randomized Study. Pediatrics. 1999;104(5):e64.

37. Kelly CP, Pothoulakis C, LaMont JT. Clostridium Difficile Colitis. N Engl J Med. 1994;330(4):257-262.

38. Gerding DN. Disease Associated with Clostridium Difficile Infection. Ann Intern Med. 1989;110(4):255-257.

39. Fekety R, Shah AB. Diagnosis and Treatment of Clostridium Difficile Colitis. JAMA. 1993;269(1):71-75.

40. McFarland LV, Surawicz CM, Greenberg RN, Elmer GW,
Moyer KA, Melcher SA, Bowen KE, et al. Prevention of Beta-Lactam-Associated Diarrhea by Saccharomyces Boulardii Compared with Placebo. Am J Gastroenterol. 1995;90(3):439-448.

41. Kotowska M, Albrecht P, Szajewska H. Saccharomyces Boulardii in the Prevention of Antibiotic-Associated Diarrhoea in Children: A Randomized Double-Blind Placebo-Controlled Trial. Aliment Pharmacol Ther. 2005;21(5):583-590.

42. Dunne C, O'Mahony L, Murphy L, Thornton G, Morrissey D, O'Halloran S, Feeney M, et al. In Vitro Selection Criteria for Probiotic Bacteria of Human Origin: Correlation with in Vivo Findings. Am J Clin Nutr. 2001;73(2 Suppl):386S-392S.

43. Pedone CA, Bernabeu AO, Postaire ER, Bouley CF, Reinert P. The Effect of Supplementation with Milk Fermented by Lactobacillus Casei (Strain Dn-114 001) on Acute Diarrhoea in Children Attending Day Care Centres. Int J Clin Pract. 1999;53(3):179-184.

44. Pedone CA, Arnaud CC, Postaire ER, Bouley CF, Reinert P. Multicentric Study of the Effect of Milk Fermented by Lactobacillus Casei on the Incidence of Diarrhoea. Int J Clin Pract. 2000;54(9):568-571.

45. Saavedra JM, Bauman NA, Oung I, Perman JA, Yolken RH. Feeding of Bifidobacterium Bifidum and Streptococcus Thermophilus to Infants in Hospital for Prevention of Diarrhoea and Shedding of Rotavirus. Lancet. 1994;344(8929):1046-1049. 